Sessions d'étude - Société canadienne d'histoire de l'Église catholique

\title{
La correspondance Langevin-Laflèche
}

\section{Nive Voisine}

Volume 34, 1967

URI : https://id.erudit.org/iderudit/1007425ar

DOI : https://doi.org/10.7202/1007425ar

Aller au sommaire du numéro

Éditeur(s)

Les Éditions Historia Ecclesiæ Catholicæ Canadensis Inc.

ISSN

0318-6172 (imprimé)

1927-7067 (numérique)

Découvrir la revue

Citer cet article

Voisine, N. (1967). La correspondance Langevin-Laflèche. Sessions d'étude -

Société canadienne d'histoire de l'Église catholique, 34, 79-86.

https://doi.org/10.7202/1007425ar

Tous droits réservés @ Les Éditions Historia Ecclesiæ Catholicæ Canadensis Inc., 1968
Ce document est protégé par la loi sur le droit d'auteur. L’utilisation des services d'Érudit (y compris la reproduction) est assujettie à sa politique d'utilisation que vous pouvez consulter en ligne.

https://apropos.erudit.org/fr/usagers/politique-dutilisation/ 


\section{La correspondance Langevin-Laflèche}

Jean-Pierre-François Langevin, premier évêque de Rimouski, et Louis-François Laflèche, deuxième évêque des Trois-Rivières, sont très proches l'un de l'autre. D'abord, leur règne épiscopal coïncide presque exactement : Mgr Langevin prend charge du nouveau diocèse de Rimouski en 1867 et est forcé de démissionner en 1890 et meurt en 1892; nommé coadjuteur de Mgr Cooke des Trois-Rivières, à la fin de 1866, Mgr Laflèche est sacré en 1867, devient administrateur du diocèse en 1869 , évêque en titre en 1870 et disparaît en 1898. Leurs idées sont aussi assez voisines : tous les deux sont conservateurs en politique, ultramontains, partisans d'une Eglise forte, adversaires du libéralisme sous toutes ses formes. Une étude des caractères nous révélerait sans doute des traits semblables, par exemple un goût de l'autoritarisme, une fermeté inflexible qui, chez Langevin du moins, pouvait tourner à la tyrannie ${ }^{1}$. Leurs contemporains - surtout les adversaires - sont d'ailleurs les premiers à les comparer; de ce point de vue, les lettres anonymes reçues par Mgr Laflèche sont révélatrices : l'une d'elles conseille à l'évêque des Trois-Rivières :

... vous êtes devenu une charge à la société et à l'Eglise ... on vous considère comme une nuisance publique... demandez un coadjuteur; on ne vous le refusera pas; on vient d'en accorder un à Mgr Langevin 2...

En 1891, Un ami dévoué et qui sait prophétise : « Le sort de Mgr Langevin vous est réservé si vous faites la moindre faute dans les prochaines élections ${ }^{3}$... »

Tant de liens communs expliquent que les évêques ont régulièrement échangé leurs vues dans une correspondance assez volumineuse. Celle-ci nous a semblé suffisamment importante pour que nous vous proposions aujourd'hui d'en analyser l'état de conservation et les principaux thèmes et de vous en donner un exemple de son contenu.

\section{Etat de conservation de la correspondance Langevin-Laflèche}

La correspondance des deux évêques a été conservée, avec plus ou moins de succès, aux deux évêchés respectifs.

A Rimouski, les archives de l'archevêché possèdent, classées dans une chemise spéciale, 31 lettres de Laflèche à Langevin. C'est très peu, si l'on songe au volume de la correspondance échangée entre les deux hommes. Les copies des lettres de Langevin à Laflèche sont plus nom-

1 Voir un portrait cruel mais réaliste de Mgr Langevin dans Andrée DésrLets, c.n.d., Un Père de la Confédération canadienne, Hector-Louis Langevin (1826-1906), thèse présentée à l'Ecole des Gradués de l'Université Laval pour obtenir le doctorat ès lettres-histoire (avril 1967), 21-24.

2 Anonyme à Mgr Laflèche, sans date, ASTR, Fonds Laflèche, B2 A2.

3 Anonyme à Mgr Laflèche, 16 fév. 1891, ibid. 
breuses : 68 dans trois registres qu'on peut intituler Correspondance spéciale, 7 autres dispersées dans les huit registres de la Correspondance générale de Mgr Langevin.

A Trois-Rivières, la correspondance Langevin-Laflèche fait partie du Fonds Laflèche, déposé aux archives du Séminaire Saint-Joseph. Une première chemise contient les lettres de 1867 à 1880; une deuxième, celles de 1881 à 1891 . Soit en tout : 105 pièces. Il s'agit des lettres originales de Langevin à Laflèche dont nous avons les copies à Rimouski (sauf

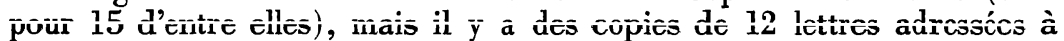
d'autres évêques. Notons également que 32 lettres de Langevin à Laflèche (dont nous avons les copies à Rimouski) n'ont pas été conservées à Trois-Rivières.

\section{Principaux thèmes de la correspondance Langevin-Laflèche}

Malgré ses lacunes, cette correspondance est extrêmement utile à la compréhension de la période 1867-1896 si fertile en discussions politico-religieuses. Presque toutes les lettres abordent l'un ou l'autre problème de l'époque.

Il s'agit, en effet, d'une correspondance très officielle. Très rarement y traite-t-on de questions strictement personnelles. En mars 1867, Langevin annonce sa nomination à Rimouski en ces termes :

Mais, Monseigneur, je connais un pauvre prêtre, dénué de santé et de vertu, sans cxpéricnce aucune de l'administration d'un diocèse, et qui vient d'être appelé à fonder un nouveau siège épiscopal, sans ressources humaines, et par conséquent à tout créer, à tout organiser. Plus que tout autre, vous sympathiserez sans doute avec moi, et vous aurez la charité de prier pour que je ne succombe pas sous ce fardeau 4 .

En 1870, Mgr Laflèche raconte son voyage de retour de Rome ${ }^{5}$. De temps en temps, de courtes lettres sont échangées à l'occasion d'une nomination, d'un heureux retour, d'un anniversaire, mais on ne peut dire qu'il y ait véritable épanchement de cœur dans des lettres privées.

Plusieurs lettres traitent de problèmes strictement administratifs. Le meilleur exemple est cette lettre de 1874 où $\mathrm{Mgr}$ Laflèche répond à une consultation sur la contestation d'une élection de marguillier ${ }^{6}$. En ce temps où le clergé est très mobile, il est très souvent question d'échange de prêtres ou d'ecclésiastiques. Ces lettres sont bien anodines, sauf quand il s'agit de fixer l'abbé Alexis Pelletier, apôtre du gaumisme au pays, ou quand Mgr Langevin demande d'urgence un prêtre pour diriger son séminaire, l'abbé Sylvain venant de donner sa démission. On découvre que les relations sont extrêmement tendues entre l'évêque de Rimouski et son séminaire et que Mgr Langevin veut profiter de l'expérience des difficultés de l'évêque des Trois-Rivières avec le séminaire de Nicolet.

4 Mgr Langevin à Mgr Laflèche, 11 mars 1867, ASTR, Fonds Laflèche,

5 Mgr Laflèche à Mgr Langevin, 6 août 1870, AAR, Lettres de Mgr Laflèche. 6 Le même au même, 4 janv. 1874, ibid. 
Au risque de passer pour un importun, je prie le nouveau V.G. d'avoir la bonté de me communiquer une copie de la décision de Rome dans les difficultés entre V.G. et le Séminaire de Nicolet. (...) Comme ici on m'a aussi contesté les mêmes droits, je considère comme de la plus haute importance pour moi et mes successeurs de connaître la décision donnée pour Nicolet 7 .

Il est également question de la division de certains diocèses (détachement de la Côte Nord, par exemple), de la formation de certains archidiocèses (Montréal, Ottawa), de l'installation de chanoines, etc.

Mais ce sont sans conteste les problèmes religieux ou politicoreligieux de l'époque qui forment la trame de cette correspondance. Dans une lettre de 1884, Langevin, en proposant à Laflèche des thèmes d'étude pour une réunion d'évêques, énumère ces questions que nous retrouvons dans la correspondance :

1. L'influence indue (pour le passé et l'avenir);

2. Le clergé de la province calomnié à Rome (évêques et prêtres);

3. Le libéralisme dans la province (passé et présent);

4. La franc-maçonnerie (ses efforts, ses progrès, ses fautes indirectes);

5. Les droits de l'Eglise et les protestants dans notre province (crainte exagérée à dessein);

6. L'Episcopat et l'Université (rapports mutuels passés et présents);

7. L'enseignement universitaire (est-il ce qu'il doit être, surtout en droit, en médecine, en histoire...? ?);

8. Le personnel universitaire (laïc et ecclésiastique);

9. L'Archevêque et les Suffragants;

10. L'Archevêque et la presse;

11. La question de l'éducation;

12. Les intrigues à Rome (les moyens de les déjouer à l'avenir) ${ }^{8}$.

Tous ces sujets ne sont pas traités de long en large; on ne fait parfois qu'une allusion à l'un ou à l'autre, mais on en parle quand même à un moment donné de la correspondance.

Il est cependant un sujet qui cristallise tous les autres et revient sans cesse dans les lettres de l'un et l'autre évêque, c'est la question du libéralisme et de l'influence indue. C'est elle qui va nous permettre de donner un exemple du contenu de la correspondance Langevin. Laflèche.

\section{Echange Langevin-Laflèche sur l'influence indue}

La correspondance Langevin-Laflèche est particulièrement forte à deux périodes : de 1883 à 1885 , au moment où Laflèche se bat contre la division de son diocèse et organise la résistance pour la visite de dom Smeulders; de 1876 à 1878, au temps de la grande querelle de l'influence indue et du procès de Bonaventure où est impliqué Mgr de Rimouski. C'est de cette dernière période seulement que nous allons maintenant vous parler.

Avant 1876 , les deux évêques échangent assez peu de lettres. En 1867, Mgr Laflèche suggère d'écrire un mandement en faveur de la

7 Mgr Langevin à Mgr Laflèche, 18 déc. 1883, AAR, Correspondance spéciale, III: $284 \mathrm{~s}$.

8 Le même au même, 13 janv. 1884, ibid., III : 289s. 
Confédération et Mgr Langevin acquiesce ${ }^{9}$. Le Concile de Vatican I crée une certaine amitié entre les deux hommes que des lettres nous rappellent. Fait assez curieux, il n'y a pas échange de vues à propos du Programme catholique de 1871 : cela peut s'expliquer non seulement par le fait que Langevin est l'ami du nouvel archevêque (libéral) Taschereau, opposé au Programme, mais aussi parce que, frère d'un ministre conservateur, il ne veut pas affaiblir le parti de Cartier-Macdonald. Comme l'Archevêque de Québec, il écrira à ses fidèles qu'il n'a pas été consulté et, par son journal local, la Voix du Golfe, il fera tirer à boulets rouges (sı on peut dire) sur İes programmıstes. Si bien que ies reiations deviennent tendues entre Mgr Langevin et Mgr Laflèche : le premier profite de deux incidents mineurs pour monter le ton

Ainsi V.G. ne se contente pas de me laisser insulter, avec son Archevêque et un autre de ses Collègues, par le ( Journal des Trois-Rivières ), au sortir même de notre réunion épiscopale; Elle vient de plus embaucher un de mes prêtres (Alexis Pelletier), avec lequel j'étais en pourparlers pour lui donner un poste. C'est vraiment une singulière manière d'entendre et de pratiquer la charité, la justice et le respect de l'ordre épiscopal ${ }^{10}$.

Heureusement des explications pacifiques de Mgr Laflèche permettent d'éloigner l'orage.

Mais l'amitié ne revient vraiment qu'avec la question de l'influence indue; on peut dire que c'est à ce moment que les deux évêques sont le plus liés. précise :

En 1874, une nouvelle loi électorale fédérale (38 Vict., 75,258 )

Quiconque, directement ou indirectement, par lui-même ou par quelqu'un d'autre, en son nom, emploie ou menace d'infliger par lui-même ou par l'entremise de tout autre personne quelque lésion, dommage, préjudice ou perte d'emploi, ou de toute manière que ce soit a recours à l'intimidation contre quelque personne pour induire cette personne à voter ou à s'abstenir de voter à une élection ..., cette personne sera considérée avoir commis le délit d'influence indue 11 .

Une question se pose : les sermons où les curés vitupèrent le libéralisme et les libéraux sont-ils de l'intimidation ou de l'influence indue ? Les victimes prétendent que oui et contestent des élections " pour influence indue du clergé ».

Deux contestations touchent Mgr Langevin de très près. D'abord celle de Charlevoix où son frère Hector a reçu un appui sûr (c'est peu dire) du clergé contre son adversaire Tremblay; mais le rapport est trop indirect pour que nous en parlions ici. Celle de Bonaventure le touche directement puisqu'elle le met en cause avec son clergé.

Rappelons brièvement les faits. Aux élections provinciales de 1875, la lutte, dans le comté de Bonaventure, se fait entre Pierre-Clovis Beau-

9 Le même au même, 25 mai 1867, ASTR, Fonds Laflèche, AI L54.

10 Le même au même, 13 nov. 1871, ibid.

11 Cité dans Noël BéLANGER, Une introduction au problème de l'influence indue, illustrée par la contestation de l'élection de 1876 dans le comté de Charlevoix, thèse présentée à l'Université Laval pour obtenir la licence ès lettres-histoire (déc. 1960), 30s. 
chesne, conservateur, et John Robinson Hamilton, marchand protestant et libéral. Immédiatement, Mgr Langevin prend peur. Il écrit au curé de Carleton:

J'apprends qu'un certain protestant parcourt le comté de Bonaventure comme candidat pour la chambre locale. Il me semble que nos intérêts les plus chers, surtout nos intérêts religieux seraient bien mal placés entre ses mains 12 .

Au curé de Grande-Rivière, il ajoute :

Je désire donc que le clergé use de son influence prudemment, mais activement pour engager nos catholiques à ne pas lui accorder leur appui, et même à l'opposer. Veuillez vous entendre avec vos confrères à ce sujet, et donnez-m'en des nouvelles 13 .

Il n'en fallait pas tant pour réveiller la ferveur antilibérale des curés de Bonaventure qui pourfendent à qui mieux mieux l'adversaire de la religion. C'est d'autant plus facile pour eux qu'une lettre pastorale de Mgr Langevin défend de voter pour les libéraux :

Et ne nous dites pas, N.T.C.F., que l'on voit parmi les partisans de ces doctrines dangereuses, dites libérales, des hommes honorables, paisibles, exemplaires! Ce sont les dupes de ceux qui les mènent ${ }^{14}$.

Dans ces conditions, Hamilton est facilement battu, mais il conteste l'élection de son adversaire. L'affaire est entendue à New-Carlisle à l'automne 1876, puis soumise, en revision, à la Cour Supérieure présidée par le juge Louis-Napoléon Casault, professeur à l'université Laval.

Le jugement, rendu le 19 décembre 1876, disqualifie les deux candidats, mais Beauchesne l'est tout spécialement pour influence cléricale indue. Mgr Laflèche réagit le premier. Dans une lettre à Langevin, il suggère déjà une action :

En présence d'une pareille prétention (que les évêques ne se sont pas prononcés contre la loi), et d'une loi susceptible d'une pareille interprétation de la part d'un juge Catholique de la Valeur de M. Casault, n'avons-nous rien à faire? Il me semble que notre silence sera une approbation tacite de toute son argumentation que je trouve fausse et contraire aux droits de l'Eglise 15 .

Il faudrait, continue Laflèche, demander un amendement à la loi; l'évêque de Rimouski devrait commencer les démarches nécessaires auprès de l'Archevêque.

Monseigneur Langevin ne manque pas de réagir violemment. Il cite le juge Casault devant le Chancelier Apostolique (Mgr Taschereau) pour "qu'il soit privé de sa chaire dans l'intérêt de la jeunesse et pour l'honneur de l'Université ${ }^{16}$ » et annonce à Laflèche

12 Mgr Langevin à F.A. Blouin, curé de Carleton, 6 avril 1875, AAR, Correspondance générale, F. $\mathrm{n}^{\circ} 854$.

13 Mgr Langevin à Pierre Saucier, curé de Grande-Rivière, 13 avril 1875, ibid., $\mathrm{F}, \mathrm{n}^{\circ} 863$.

14 Mgr Jean Langevin, Lettre pastorale, 28 mai 1875, citée dans Béatrice Chassé, L'affaire Casault-Langevin, thèse présentée à l'Ecole des Gradués de l'Université Laval pour obtenir le diplôme d'études supérieures en histoire (mars 1965), 9.

Laflèche.

15 Mgr Laflèche à Mgr Langevin, 23 déc. 1876, AAR, Lettres de $\mathrm{Mgr}$ op. cit., 36.

16 Mgr Langevin à Mgr Taschereau, 4 janv. 1877, cité dans Chassé, 
...si je n'obtiens pas entière satisfaction, (je vais en référer) au Souverain Pontife lui-même. (...) Je pense aussi publier une Lettre Pastorale pour condamner ces principes si faux et si contraires aux droits et aux libertés de l'Eglise. (...)

Je préférerais encore une Lettre collective, signée au moins de tous les Suffragants, si l'Archevêque refuse de se joindre à nous ${ }^{17}$.

Laflèche est d'accord, mais il doute que Taschereau veuille signer une lettre collective.

Si Monseigneur l'Archevêque refuse son concours, je pense qu'il vaudrait mieux alors que V.G. seule adressât cette lettre à son diocèse et que les autres suffragans (sic) y donnassent leur adhésion en la publiant chacun dans leurs diocèses. C'est assez souvent ce qui se fait en France ${ }^{18}$.

Laflèche voit juste : Taschereau renvoie la cause à Rome, ce qui lui attire l'ire de Langevin et une lettre "privée » presque impertinente :

Je crois avoir rempli un devoir envers V.G. en l'avertissant de ce qui se pense et se dit à propos des griefs contre certains professeurs de l'U. $L$. Votre G. paraît s'en être choquée, j'espère ne plus mériter le reproche d'abuser de ma vieille amitié pour lui faire entendre la vérité. Dorénavant je me tiendrai dans les bornes qu'impose la position d'un Suffragant à l'égard de son Métropolitain. Si je les ai dépassées, c'est que je m'y croyais autorisé par une certaine lettre de l'Archevêque actuel de Q. lors de sa nomination, mais je l'ai mal comprise, et je ne m'y ferai plus prendre 19 .

Langevin décide alors de s'adresser à Rome et de publier un Mandement dans son diocèse. Celui-ci paraît le 15 janvier 1877 : il est un véritable pamphlet contre le jugement Casault et énumère cinq propositions totalement condamnables : "Nous déclarons indignes des sacrements ceux qui soutiendraient ces propositions ou aucune d'elles jusqu'à ce qu'ils les aient désavouées ${ }^{20}$.

\section{Laflèche est le premier à le féliciter :}

... Cet acte courageux de votre part va soulager grandement la conscience d'un bon nombre de véritables Catholiques qui ne manquent pas de bénir le Bon Dieu en voyant la vérité affirmée aussi catégoriquement en face de l'erreur officielle. J'ai la confiance que vos Collègues ne manqueront pas de donner leur adhésion à ce document important, et qu'il en résultera un grand bien 21 .

C'est d'ailleurs grâce à l'entremise de l'évêque des Trois-Rivières qu'une approbation signée par trois collègues (Laflèche, Duhamel d'Ottawa, Moreau de Saint-Hyacinthe) est envoyée à Mgr Taschereau. C'est peu et Mgr Langevin en est très ulcéré. Il ne craint pas de juger sévèrement certains évêques qui ont flanché, particulièrement Mgr Racine de Sherbrooke.

Celui qui me surprend le plus, c'est Mgr de Sherbrooke: s'est-il fait acheter? Un revirement si subit et si complet me semble inexplicable AI L54.

17 Mgr Langevin à Mgr Laflèche, 29 déc. 1876, ASTR, Fonds Laflèche,

is Mgr Laflèche à Mgr Langevin, 3 janv. 1877, AAR, Lettres de .Mgr Laflèche.

19 Mgr Langevin à Mgr Taschereau, 16 janv. 1877, AAR, Correspondance spéciale, III : 214 .

20 Cité dans Chassé, op. cit., 44s.

Laflèche.

21 Mgr Laflèche à Mgr Langevin, 21 janv. 1877, AAR, Lettres de Mgr 
autrement. Sous ces circonstances, je ne puis consentir à le constituer mon agent à Rome pour nos affaires, excepté pour présenter l'adresse de la Province au Pape. Pour tout le reste, je n'ai plus confiance en lui : il nous trahit. J'en ai des preuves 22 .

Laflèche n'en est pas moins choqué et il n'est pas loin de lancer des imprécations.

... Bon Dieu ! que de misères dans la défense de la vérité ! Ce qui me gêne le plus dans tout cela c'est la recommandation que l'on m'a répétée bien des fois à Rome, de l'Union entre les Evêques ? Pourtant il eût été si facile d'être unis si Mgr l'Archevêque avait voulu prendre l'initiative ! Faut-il donc que le jugement d'un seul l'emporte sur celui de tous les autres ? et n'y a-t-il d'accord possible que dans le silence? En attendant l'erreur ne demande pas mieux et elle fait son chemin! Fermer la bouche aux Prêtres, aux Evêques, et même au Pape s'il en fallait croire les derniers journaux, tel semblerait être le mot d'ordre pour le moment 23 .

Langevin ne se sent pas moins seul et Laflèche est obligé de le consoler.

Ne vous affligez point de l'isolement où V.G. s'est trouvée dans la condamnation du jugement Casault; cet acte de fermeté vraiment épiscopal sera assurément l'une des belles pages de votre épiscopat et déjà les hommes les plus capables de le bien apprécier en font le plus bel éloge $24 .$. .

La réponse de Rome fut l'envoi d'un délégué, Mgr Conroy. Mgr Langevin l'attend avec réticence, car, écrit-il à Laflèche, " on semble l'attendre assez prochainement en compagnie de $M g r$ Pâquet ${ }^{25}$ ". Nous n'avons pas les lettres de Mgr Laflèche pendant cette période, mais celles de Langevin indiquent qu'il veut pousser les prêtres et les laïcs à présenter des mémoires au délégué.

Ne jouons pas ici le rôle de dupes, en laissant nos libéraux prendre les devants et circonvenir le Délégué. Tenons-nous prêts et ne permettons pas qu'ils nous ferment encore une fois la bouche, sous prétexte que la chose pourrait déplaire à certain personnage 26 .

Lui-même publie une lettre circulaire le 29 septembre 1877 et s'adresse directement au délégué. Peine perdue ! La réponse de la Congrégation de la Propagande est formelle : le juge Casault peut demeurer professeur à Laval ${ }^{27}$.

C'est une défaite totale pour l'évêque de Rimouski. Il ne l'accepte pas facilement; à son confident des Trois-Rivières, il écrit :

Je serais prêt à aller plus loin et à dire que nos libéraux ont réussi à tromper le Délégué Apostolique, comme ils ont trompé la Propagande et le Saint Office : ils l'ont induit à incriminer et à blâmer les hommes les plus zélés pour la défense des droits de l'Eglise, à relever et à faire

22 Mgr Langevin à Mgr Laflèche, 2 fév. 1877, ASTR, Fonds Laflèche, AI L54.

23 Mgr Laflèche à Mgr Langevin, 5 fév. 1877, AAR, Lettres de Mgr Laflèche.

24 Le même au même, 15 avril 1877, ibid.

25 Mgr Langevin à Mgr Laflèche, 13 mars 1877, AAR, Correspondance spéciale, I, no 255.

26 Le même au même, 22 avril 1877, ASTR, Fonds Laflèche, AI L54.

27 Pour le détail, voir Chassé, op. cit., 50-115. 
triompher ceux qui les ont attaqués le plus impudemment, et à lui inspirer une peur excessive des protestants et des hommes au pouvoir 28 .

Les deux évêques continuent à se concerter pour justifier, par un mémoire, le clergé devant le Saint-Office et pour demander l'amendement de la loi électorale. Mais plus rien ne marche et, malheur suprême, les libéraux prennent le pouvoir (1878).

Voilà nos Libéraux rendus au pouvoir dans notre pauvre Province ! Nous allons en voir de belles! S'ils réussissent à se maintenir, ils en opèreront des réformes à leur façon! Avant deux ans, ils auront arraché le conirôle de i'éducation au Clergé é aboii le Conseil de l'Insiruciion Publique, pour tout conduire à leur gré. (...) ....Mon cher Seigneur, nous nous en allons à la Révolution, et grand train! Le Délégué et l'Archevêque en auront été les premières et principales causes; le Clergé en sera la première et principale victime. C'est là ma conviction 29 .

Cette escalade de malheurs semble émousser l'amitié des deux évêques : dans les années qui suivent, les lettres deviennent de plus en plus rares. Y a-t-il un froid entre eux ? Deux lettres de Mgr Langevin à Mgr Taschereau le laisseraient supposer.

Il me semble (...) que Mgr des Trois-Rivières devrait être réduit à se borner au gouvernement de son propre diocèse, sans se mêler des affaires de ses voisins et sans se faire le centre de tous les mécontents 30 . Est-ce que personne ne répondra à $\mathrm{Mgr}$ Laflèche au sujet des Ecoles Normales? Il pourrait bien en demander une, sans attaquer les autres ainsi sans mesure et sans justice 31 .

C'est quand même une éclipse plutôt courte, puisque, dès 1882-1883, la correspondance reprend de plus belle et que Mgr Langevin donne son appui à Laflèche qui lutte pour conserver intact son diocèse.

Cette correspondance, dont nous n'avons pu donner qu'un aperçu bien succinct, éclaire donc d'une façon particulière beaucoup des problèmes du $\mathrm{XIX}^{\bullet}$ siècle; elle permet aussi de déceler tout un monde d'intrigues et de combines dans l'Eglise de cette époque. Analysée plus profondément, elle nous révélerait bien des traits de caractère de ces évêques qui ont quand même joué un rôle important à la fin du siècle dernier. Nous souhaitons pour bientôt des études approfondies sur ces matériaux qui dorment dans les archives diocésaines.

Nive VoIsIne,

Collège de Rimouski.

\footnotetext{
AI L54.

29 Le même au même, 14 mars 1878, ibid.

30 Mgr Langevin à Mgr Taschereau, 11 juin 1881. AAR, Correspondance spéciale, III : 29.

31 Le même au même, 7 sept. 1881, ibid., III : 44 .
}

28 Mgr Langevin à Mgr Laflèche, 26 janv. 1878, ASTR, Fonds Laflèche, 